\title{
General Guidelines in the Management of an Obstetrical Patient on the Labor and Delivery Unit during the COVID-19 Pandemic
}

\author{
Angela J. Stephens, MD ${ }^{1}$ John R. Barton, MD ${ }^{2}$ Nana-Ama Ankumah Bentum, MD ${ }^{1}$ \\ Sean C. Blackwell, MD ${ }^{1}$ Baha M. Sibai, MD ${ }^{1}$
}
${ }^{1}$ Department of Obstetrics, Gynecology, and Reproductive Sciences, McGovern Medical School, The University of Texas Health Science Center at Houston, Houston, Texas
2 Division of Maternal-Fetal Medicine, Baptist Health Lexington, Lexington, Kentucky

\begin{abstract}
Address for correspondence Angela J. Stephens, MD, Department of Obstetrics, Gynecology, and Reproductive Sciences, University of Texas Health Science Center at Houston, 6431 Fannin Street, MSB 3.270, Houston, TX 77030 (e-mail: angela.j.stephens@uth.tmc.edu).
\end{abstract}

Am J Perinatol 2020;37:829-836.

\begin{abstract}
Keywords

- coronavirus

- COVID-19

- pandemic

- labor and delivery

Novel coronavirus disease 2019 (COVID-19) is a respiratory tract infection that was first identified in China. Since its emergence in December 2019, the virus has rapidly spread, transcending geographic barriers. The World Health Organization and the Centers for Disease Control and Prevention have declared COVID-19 as a public health crisis. Data regarding COVID-19 in pregnancy is limited, consisting of case reports and small cohort studies. However, obstetric patients are not immune from the current COVID-19 pandemic, and obstetric care will inevitably be impacted by the current epidemic. As such, clinical protocols and practice on labor and delivery units must adapt to optimize the safety of patients and health care workers and to better conserve health care resources. In this commentary, we provide suggestions to meet these goals without impacting maternal or neonatal outcomes.
\end{abstract}

Key Points

- Novel coronavirus disease 2019 (COVID-19) is a pandemic.

- COVID-19 impacts care of obstetric patients.

- Health care should be adapted for the COVID-19 pandemic.

The novel coronavirus disease 2019 (COVID-19) is a rapidly disseminating respiratory tract infection that was first identified in the Hubei province of central China. ${ }^{1-4}$ Since its emergence in December 2019, COVID-19 has transcended barriers of geography, age, race, and sex. In January 2020, the World Health Organization (WHO) declared that the epidemic is a public health emergency of international concern. ${ }^{1,3}$

With the increase in reported cases, COVID-19 has become increasingly relevant to obstetricians. ${ }^{5,6}$ However, there is little data regarding COVID-19 in pregnancy. ${ }^{6-9}$ In the absence of strong data, it is important to make decisions on clinical judgment and with common sense. In this commentary, we suggest practical changes to prepare for the potential effects of the COVID-19 pandemic on our health care resources, particularly on labor and delivery. The goals of these recommendations are two folds: (1) to minimize hospital length of stay and (2) to minimize the exposure of potentially infected patients to health care workers. received

April 9, 2020

accepted after revision

April 11, 2020

published online

April 28, 2020
Copyright $\odot 2020$ by Thieme Medical Publishers, Inc., 333 Seventh Avenue, New York, NY 10001, USA. Tel: +1(212) 760-0888.
DOI https://doi.org/

10.1055/s-0040-1710308. ISSN 0735-1631. 
The following are recommendations to achieve the above desired goals in the obstetrical population without adversely affecting maternal or perinatal outcomes. These recommendations are not made in a vacuum but reflect input from experts across the country and a review of current literature.

\section{Background on Coronavirus}

Coronaviruses are single-stranded RNA, enveloped viruses. ${ }^{10}$ They are transmitted primarily through respiratory droplets and direct contact. ${ }^{3,4,10}$ Diagnosis of COVID-19 is primarily through viral nucleic acid detection using real-time polymerase chain reaction (RT-PCR). Serology as a diagnostic procedure should be used only if RT-PCR is not available. ${ }^{5}$ Other diagnostic signs include lymphopenia, elevated C-reactive protein, elevated liver transaminases, and radiographic findings consistent with pneumonia. ${ }^{7,8,11,12}$ COVID-19 has variable clinical presentations. ${ }^{1,5}$ Symptoms are typically mild and may include fever, fatigue, anorexia, malaise, myalgias, headache, cough, rhinorrhea, nasal congestion, sore throat, shortness of breath, nausea, emesis, or diarrhea. ${ }^{4,5}$ Approximately $14 \%$ of affected individuals develop severe disease requiring hospitalization. ${ }^{4}$

Theoretically, pregnant women may be more susceptible to general and severe COVID-19 morbidity due to physiologic and immunologic changes in pregnancy as evidenced by experiences with other coronaviruses. ${ }^{5,6,13,14}$ Case reports and cohorts of pregnant women with COVID-19 typically involve mild symptoms such as fever or cough.,11,13-15 An association between COVID-19 in pregnancy and preterm rupture of membranes, preterm delivery, intrapartum fetal heart rate abnormalities, such as tachycardia, and cesarean delivery are also suggested by limited publications. ${ }^{2,5-8,11,15,16}$ Zeng et al reported three neonates from a cohort of 33 patients who may have been infected with severe acute respiratory syndromecoronavirus-2 (SARS-CoV-2) in utero from mothers with COVID-19. ${ }^{17}$ Evidence for transmission was based on elevated immunoglobulin (Ig)-M antibody values in blood drawn from the neonates following birth. No infant specimen had a positive reverse transcriptase-polymerase chain reaction test result; therefore, there was no virologic evidence for congenital infection in these cases to support the serologic suggestion of in utero transmission. ${ }^{17}$ A second study of six known COVID-19positive mothers and their infants showed similar results. ${ }^{18}$ However, several other studies have not demonstrated any evidence of vertical transmission of COVID-19. 5,7,8,19-21

There are no current coronavirus-specific vaccines or therapeutics available. ${ }^{1,5}$ In the absence of vaccines or targeted therapy, management of COVID-19 in pregnancy must focus on prevention. ${ }^{22,23}$ Pregnant women should be advised to avoid unnecessary travel, group events, public transportation, and contact with symptomatic individuals. ${ }^{5}$

\section{General Considerations on Labor and Delivery during COVID-19 Pandemic}

Screen All Patients and Visitors on Labor and Delivery All patients presenting for triage or scheduled delivery should be screened for symptoms of COVID-19 by staff at time of presentation to labor and delivery. The number of entry points to labor and delivery should be limited to ensure all patients and visitors are screened. Women should be evaluated for signs or symptoms of COVID-19. ${ }^{4,5}$ If a patient calls the hospital prior to arrival, screening should occur over the phone to best prepare and allocate resources prior to the patient's arrival.

\section{Limit Visitors on Labor and Delivery}

Current data suggest that person-to-person transmission most commonly occurs during close contact with infected individuals, primarily through respiratory droplets. ${ }^{22}$ Not all individuals infected are symptomatic, and therefore may not be identified through screening upon entry to the hospital or the labor and delivery unit. Therefore, there should be limited visitation on labor and delivery. No visitors should be allowed for persons under investigation (PUI) or COVID-19-positive patients. For all other patients on labor and delivery during the current pandemic, institutions could consider one visitor at time of delivery. However, for those areas of the country with a significant COVID-19 burden, a universal no visitation policy may be more advantageous.

\section{Isolate PUls or COVID-19-Positive Patients}

Women with symptoms suggestive of COVID-19 should be immediately isolated. For those with confirmed testing, patients should be placed in a negative pressure room. If negative pressure rooms are not available, then patients should be placed in isolated rooms away from other occupied rooms. Isolation room filtration systems, such as the high-efficiency particulate absorbing (HEPA) units, can also be utilized to create portable, negative pressure rooms when options are otherwise limited. ${ }^{24-26}$ Movement of both PUIs and confirmed positive patients should be limited throughout the facility. Personnel involved in patient care should be limited to only those necessary.

\section{Use Personal Protective Equipment with PUls or COVID-19-Positive Patients}

Standard respiratory precautions should be utilized in all areas of labor and delivery by providers, patients, and visitors. All health care professionals caring for PUIs or confirmed COVID-19-positive patients should use appropriate personal protective equipment (PPE). Droplet and contact precautions (including face shield or goggles, surgical mask, gown, and gloves) should be used for all clinical interactions in agreement with Centers for Disease Control and Prevention (CDC) and World Health Organization (WHO) guidelines. $^{3,22}$ N95 masks should be used whenever an aerosolizing procedure is being performed or likely to be performed on a patient with suspected or confirmed COVID19 such as cesarean delivery, postpartum hemorrhage requiring transfer to the operating room, or intubation. ${ }^{22,27}$ All patients and visitors should be encouraged to wear surgical masks at all times, but particularly those with suspected or confirmed infection. 


\section{Delivery Planning on Labor and Delivery during COVID-19 Pandemic}

\section{Creation of Designated Delivery Locations for PUls or CoviD-19-Positive Patients}

A designated room with negative pressure capabilities should be reserved for PUIs or COVID-19-positive patients. A negative pressure operating room for pregnant women who warrant cesarean delivery with suspected or confirmed infection should also be established in advance of need. Special consideration should also be made to ensure there is a designated isolation room for newborns of mothers with suspected or confirmed infection in close proximity of delivery or operating room. If negative pressure rooms are not available, then patients should be placed in isolated rooms away from other occupied rooms. HEPA units can also be considered for the prevention of the spread of airborne viral organisms. ${ }^{24-26}$

\section{Mode of Delivery}

Mode of delivery should continue to be individualized based on obstetric indications.

\section{Continue to Utilize Scheduled Cesarean Deliveries}

As long as there is an appropriate indication and it is performed at the appropriate gestational age, scheduled cesarean deliveries should not be delayed based on the COVID-19 pandemic. This procedure efficiently moves the patient through the delivery process allowing the patient and her newborn to be discharged in a timely fashion. Consideration can be given to day 2 postpartum discharge if the patient is stable and has adequate home resources. If a patient who is COVID-19-positive has a scheduled cesarean delivery for unrelated medical indications, a negative pressure operating room should be utilized as described above. If a PUI or COVID-19-positive patient is requiring medical treatment due to COVID-19 complications, the cesarean delivery may need to be postponed to optimize respiratory status prior to procedure. The mother should always be stabilized prior to delivery. Anesthesia, maternal-fetal medicine, and neonatology should be involved in the preoperative planning of any PUI or COVID-19-positive patient to ensure all necessary resources are available in advance.

\section{Review of Nonmedically Indicated Inductions (Elective)}

There should be some consideration of delaying elective inductions with a poor Bishop's score. While the cesarean section rate was not increased with induction at 39 weeks in A Randomized Trial of Induction Versus Expectant Management (ARRIVE), it did establish that time to delivery and length of stay were increased. ${ }^{28}$ An extended induction, even with successful vaginal delivery, increases exposure time for both patients, and staff. Medically or obstetrically indicated inductions, however, should not be delayed.

\begin{tabular}{l} 
Table 1 Potential indications for delivery with severe \\
COVID-19 infection \\
\hline Maternal indications \\
Intrauterine infection \\
Disseminated intravascular coagulation \\
Hepatic or renal failure \\
Compromised cardiopulmonary function due to uterine \\
size or peritoneal fluid \\
Compartment syndrome \\
Severe adult respiratory distress syndrome or barotrauma \\
Cardiopulmonary arrest \\
Fetal Indications \\
Fetal demise \\
Gestational age associated with low neonatal morbidity or \\
mortality
\end{tabular}

Abbreviation: COVID-19, novel coronavirus disease 2019.

${ }^{a}$ Secondary to multifetal gestation, macrosomia, or polyhydramnios.

\section{Decision for Delivery in Patients with Severe COVID-19 Infection in the Third Trimester}

The decision for delivery in the setting of severe COVID-19 infection is challenging. The increased oxygen consumption and reduced functional residual capacity of pregnancy predisposes to rapid deterioration of both maternal and fetal status when combined with the physiologic changes associated with severe infection. The decision for delivery should take into consideration gestational age, maternal status, and fetal status. Importantly, stabilizing the mother prior to emergent delivery for fetal indications is imperative. Through stabilization of the mother, the fetus' status will likely improve. ${ }^{29}$ Clinical scenarios for which delivery is likely indicated to maximize resuscitative efforts in cases of severe COVID-19 complicated by sepsis and septic shock, are presented in - Table 1. Of note, uterine distention secondary to multifetal gestations, macrosomia, and polyhydramnios among other processes can result in compromised cardiopulmonary function due to diaphragm displacement. In such patients with compromised cardiopulmonary dysfunction that requires intubation, delivery at 32 weeks should be considered and decision balanced with benefits of pregnancy continuation following consultation with neonatology.

\section{Conservative Management of PPROM after 32 to 34 Weeks of Gestation}

The potential benefits of additional in utero time for preterm prelabor rupture of membranes (PPROM) must be balanced in light of current COVID-19 pandemic circumstances and known adverse outcomes in patients with PPROM such as chorioamnionitis, placental abruption, cord prolapse, cord compression, or uteroplacental insufficiency. ${ }^{30}$ Despite the recent randomized control trial of singletons between $34^{0 / 7}$ and $36^{6 / 7}$ weeks with PPROM by Morris et al demonstrating no increased risk of neonatal sepsis or composite neonatal morbidity (sepsis, mechanical ventilation $\geq 24$ hours, stillbirth, or neonatal death), in the current setting, there are additional risks associated with 
extended hospital stays to be considered for patients. ${ }^{31}$ Additionally, this trial also demonstrated increased maternal morbidity, such as hemorrhage and infection, associated with expectant management of PPROM after 34 weeks that would further increase exposure to different medical personnel, movement between different hospital settings (i.e., labor, operating room, and intensive care unit), and overall length of stay. ${ }^{31}$ As median latency following PPROM is $<7$ days, particularly after 29 to 30 weeks, the potential benefits of expectant management beyond 34 weeks may be mitigated by the development of associated adverse outcomes and continued hospitalization during the COVID-19 pandemic. $^{31-33}$ A case could also be made for induction at 32 weeks of gestation based on the perinatal outcomes from previous studies following consultation with neonatology. ${ }^{34-36}$ Pasquier et al conducted a randomized control trial of 139 women with PPROM between $28^{0 / 7}$ and $31^{6 / 7}$ weeks and allocated them to either expectant management with delivery at 34 weeks or delivery 24 hours after completion of steroid course. ${ }^{35}$ This study found no significant differences in neonatal outcomes between groups. A second randomized control trial conducted by Cox and Leveno of 129 women with PPROM between 30 and 34 weeks had similar results. $^{36}$

\section{Intrapartum Management}

\section{When Possible, Limit Frequency and Duration of Room Visits and Number of Cervical Exams}

As the primary risk factor for infection with COVID-19 is close contact with infected individuals, limiting frequency and duration of exposure is key in prevention for medical professionals. ${ }^{3,21}$ This involves limiting unnecessary time in rooms and reducing the number of digital cervical exams (by both nurses and physicians) when possible. For uncomplicated labor patients, exams should be spaced to limit the number of providers in direct contact with patients. Ultrasound assessments should be limited and utilized only when likely to alter clinical management.

\section{Shortening the Second Stage of Labor}

Although there has been a recent trend to allow a longer second stage of labor in hope of reducing the cesarean delivery rate due to failure to progress in the second stage, a prolonged second stage may be ill advised during the COVID-19 pandemic due to an increased risk of respiratory secretion exposures to visitors and medical personnel. ${ }^{37}$ As such, it may be prudent to resume previous recommendations of a 1 -hour second stage in a multiparous patient without an epidural ( 2 hours with an epidural) and a 2-hour second stage in a nulliparous patient without an epidural ( 3 hours with an epidural). Consideration should also be given to shortening the second stage with an operative vaginal delivery in those patients that meet prerequisites including a fully dilated cervix, fetal head engagement, low fetal station, adequate clinical pelvimetry, and patient consent.

\section{When Possible, Do Not Encourage Active Pushing During Second Stage of Labor}

If possible, active pushing during the second stage of labor should be minimized. Deep breathing and maternal expulsive efforts may increase visitor and personnel exposure to the patient's respiratory secretions. The pushing process, therefore, potentiates the risk of transmission to those in the room, particularly the nurses and physicians. As such, every effort should be made to "labor down" patients as much as feasibly possible. However, the patient should not be discouraged to push if she has a strong urge to push.

\section{Avoidance of Delayed Cord Clamping}

As previously discussed, two recent cohort studies suggest the possibility of COVID-19 vertical transmission. ${ }^{17,18}$ While several other studies have not shown these results, we must remain cautious without definitive data. ${ }^{5,7,8,19-21}$ As such, we recommend avoidance of delayed cord clamping given the potential increased risk of viral transmission to the newborn.

\section{Avoid Aggressive Fluid Hydration}

Individuals on labor and delivery, particularly PUIs or confirmed COVID-19-positive cases, should be cautiously treated with intravenous (IV) fluids. Aggressive hydration can worsen oxygenation status. ${ }^{4}$ Further, although normal saline and lactated Ringer's are excellent choices for IV fluid in a patient for which delivery is anticipated, the additional sodium load can predispose patients to pulmonary congestion. If IV fluids are required in a patient for which delivery is not anticipated, IV fluids with reduced sodium load (1/4-normal saline or $1 / 2$-normal saline) should be considered.

\section{Avoid Use of Maternal Oxygen Therapy for Fetal Resuscitative Purposes}

Several studies have demonstrated that the use of oxygen face and nonrebreather masks can cause aerosolization of respiratory secretions, thereby increasing viral transmission. ${ }^{38-40}$ On labor and delivery, maternal oxygen supplementation has historically been used for intrapartum resuscitation of nonreassuring fetal heart rate tracings. However, available evidence has not suggested any neonatal benefit to intrapartum maternal oxygen supplementation. ${ }^{41,42}$ Rather, some trials have suggested risks to the neonate including prolonged resuscitation and acidemia. ${ }^{43-45}$ In light of the increased risk of viral transmission and lack of proven fetal benefit of maternal oxygenation for fetal resuscitation, we suggest use of maternal oxygen therapy for treatment of maternal hypoxia and limiting its use for fetal resuscitation. We recognize that this may be a difficult change. Therefore, we suggest a physician review the fetal heart rate tracing prior to administration of supplemental oxygen therapy in all patients. Maternal oxygen supplementation should be reserved for patients with fetal bradycardia, prolonged, recurrent, or persistent decelerations, or other situations with a severely abnormal fetal heart rate tracing with the suspected hypoxia. 


\section{Limited Use of Antenatal Corticosteroids beyond 34 Weeks or Rescue Dosing}

For women $<34^{0 / 7}$ weeks who are candidates for an initial course of antenatal corticosteroids (ACS), we should follow current practices and administer ACS as indicated. ${ }^{46}$ However, use of ACS after 34 weeks or as a rescue course should be reconsidered during the current COVID-19 pandemic. Data regarding the benefits of late preterm steroids remain mixed. Some studies support improved respiratory outcomes for infants administered steroids in the late preterm period, while others do not show these improved outcomes and instead demonstrate an increased risk of adverse outcomes, such as neonatal hypoglycemia and sepsis. ${ }^{47-51}$ Data regarding rescue course of steroids is similarly inconsistent. ${ }^{52-57}$ Given these inconsistencies regarding perinatal benefit to late preterm and rescue ACS, as well as the suppression of the maternal immune response associated with steroid administration, we recommend all late preterm antenatal steroids and rescue courses to be withheld during the pandemic. ${ }^{5}$ For PUIs or COVID-19-positive women, we should evaluate the risk and benefits of any steroid administration on a case-bycase basis. For these individuals, the risk/benefit ratio for ACS would suggest the benefits do not seem to outweigh the risks of facilitating or worsening COVID-19 infection. Patients with unstable conditions including but not limited to eclampsia, pulmonary edema, acute respiratory distress syndrome or barotrauma, disseminated intravascular coagulation, suspected abruptio placentae, or nonreassuring fetal heart rate tracings should be delivered regardless of the benefit of corticosteroids following maternal stabilization. However, the decision to deliver should be individualized with involvement of maternal-fetal medicine, intensivists, and neonatology.

\section{Be Judicious in Magnesium Sulfate Use on Labor and Delivery}

Magnesium sulfate is a widely used medication on labor and delivery. It is usually recommended for neuroprotection for extremely premature fetuses ( $<32$ weeks) and for seizure prophylaxis in patients with preeclampsia. ${ }^{58,59}$ However, magnesium is not without risks. One major, albeit uncommon, side effect is respiratory depression. This can have serious implications for maternal outcomes, particularly for PUIs and known COVID-19-positive patients with an already compromised respiratory status. As such, the benefits of magnesium sulfate for neuroprotection and seizure prophylaxis should be weighed against the risks to the maternal respiratory function in light of gestational age and maternal respiratory status. The Society for Maternal Fetal Medicine (SMFM) has suggested that a single 4-g bolus dose of magnesium sulfate may serve as an alternative to usual dosing in the setting of mild respiratory distress with caution of any use in those with moderate-to-severe respiratory distress. ${ }^{23}$ This is appropriate in the case of impending delivery in pregnancies at $<31$ weeks. For patients with preeclampsia without severe features or gestational hyper- tension, magnesium sulfate therapy should not to be administered. In those with severe features, however, magnesium sulfate prophylaxis is indicated. As suggested by the results of the Magpie trial, an IV loading dose of 4-g magnesium sulfate over 15 to 20 minutes followed by an IV maintenance dose of $1 \mathrm{~g} / \mathrm{h}$ could be used rather than a traditional higher loading dose $(6 \mathrm{~g})$ or maintenance dose $(2 \mathrm{~g} / \mathrm{h}) .^{60}$

\section{Clinical Trials during the COVID-19 Pandemic}

Providing informed consent for randomized trials requires extensive patient interactions with research and clinical staff. For studies that are not directly related to the treatment or management of COVID-19, a delay in studies until resolution of the current pandemic may be prudent.

\section{Postpartum NSAID Use for PUls or COVID-19-Positive Patients}

There is limited data regarding use of nonsteroidal antiinflammatory drugs (NSAIDs) in the COVID-19 population. ${ }^{61,62}$ Some reports have suggested NSAIDs may exacerbate symptoms of COVID-19. This is largely based on data regarding other respiratory infections. ${ }^{61,63}$ The primary mechanism through which NSAIDs are hypothesized to impact COVID19-positive patients is through inhibition of cyclooxygenases. ${ }^{63}$ However, there remains no evidence that NSAIDs worsen disease outcomes in COVID-19 patients. ${ }^{61,62,64,65}$ Further, some studies have actually suggested a benefit to NSAID use. ${ }^{62,66}$ A 2006 study demonstrated that indomethacin may actually have antiviral activity against the SARS-CoV. ${ }^{66}$ Given the lack of conclusive data regarding NSAID use in COVID-19, as well as limited analgesic options postpartum, we recommend continued use as clinically indicated.

\section{5-Methyl Prostaglandin $F_{2 \alpha}$ Use in PUI or COVID-19-Positive Patients}

The 15-Methyl prostaglandin $F_{2 \alpha}$ is a commonly utilized uterotonic for postpartum hemorrhage. ${ }^{67}$ It should be used cautiously in patients with suspected or confirmed COVID19 infection due to its ability to increase pulmonary vascular resistance. It is therefore contraindicated in patients with active respiratory disease, such as those with severe COVID19 infection. $^{67}$

\section{VTE Prophylaxis in PUI or COVID-19-Positive Patients}

For COVID-19-positive patients or PUIs undergoing inpatient evaluation and treatment, venous thromboembolism (VTE) prophylaxis should be considered. These patients have risk factors for VTE including limited ambulation, pregnant or postpartum status, and infection among other individualized risk factors. As the clinical presentation of pulmonary embolism can mimic respiratory distress associated with severe COVID-19 infection, this should be considered in the 
differential of PUIs, particularly those with hypoxia. Unless otherwise medically indicated, VTE prophylaxis can be discontinued for PUIs or COVID-19-positive patients upon discharge.

\section{Call for a Cautious Approach to Care on Labor and Delivery}

In the wake of the COVID-19 pandemic, health care professionals must seek ways to adapt their clinical practice to protect patients, themselves, and health care resources. While the health care system in the United States has minimized elective encounters and procedures at large, obstetricians are in a unique position. The birthing process cannot be considered elective. As such, labor and delivery cannot be scaled down in the same manner as other units within the hospital. However, through thoughtful, deliberate planning and feasible changes in approach to care, we can make a difference in both exposure of potentially infected patients to health care workers and in reducing length of stay without impacting perinatal outcomes.

Despite the limited data regarding COVID-19 in pregnancy, it is important to make changes in clinical practice that consider the current health care climate. It is our belief that implementation of the above recommendations will protect patients and health care workers while also conserving health care resources.

Following are our recommendations summarized:

- Screen and limit all patients and visitors on the labor and delivery unit.

- Health care workers should use appropriate PPE on labor and delivery units.

- PUIs and COVID-19-positive patients should be isolated in compliance with CDC guidelines.

- Scheduled cesarean deliveries and medical inductions should not be delayed.

- Scheduling elective inductions with a poor Bishop's score at 39 weeks should be reconsidered.

- We should limit the frequency and duration of room visits and cervical exams during uncomplicated labor.

- Efforts should be made to shorten the second stage of labor.

- Active pushing during the second stage of labor should not be encouraged.

- Delayed cord clamping should be avoided until additional information is available regarding vertical transmission of COVID-19.

- Avoid aggressive fluid hydration and maternal oxygen therapy during labor.

- Maternal and fetal status should be balanced with clinical circumstances to make an individualized decision for delivery in a severe COVID-19-positive patient during the third trimester.

- Expectant management of PPROM after $32^{0 / 7}$ to $34^{0 / 7}$ weeks should be avoided, if possible.

- Use of late preterm and rescue antenatal corticosteroids should be limited.
- The respiratory status of patients should be considered prior to administration of magnesium sulfate.

- Suspension of clinical trials pending resolution of the current pandemic should be considered.

- NSAIDS should be used as clinically indicated postpartum.

- 15-Methyl prostaglandin $\mathrm{F}_{2 \alpha}$ should be avoided in PUIs and COVID-19-positive patients.

- Consider VTE prophylaxis in PUI or COVID-19-Positive Patients.

Conflict of Interest

None declared.

\section{References}

1 Rasmussen SA, Smulian JC, Lednicky JA, Wen TS, Jamieson DJ. Coronavirus Disease 2019 (COVID-19) and pregnancy: what obstetricians need to know. Am J Obstet Gynecol 2020 (e-pub ahead of print). Doi: 10.1016/j.ajog.2020.02.017

2 Zhu H, Wang L, Fang C, et al. Clinical analysis of 10 neonates born to mothers with 2019-nCoV pneumonia. Transl Pediatr 2020;9 (01):51-60

3 World Health Organization. Clinical management of severe acute respiratory infection (SARI) when COVID-19 disease is suspected. Available at: https://www.who.int/publications-detail/clinicalmanagement-of-severe-acute-respiratory-infection-when-novelcoronavirus-(ncov)-infection-is-suspected. Accessed April 14, 2020

4 Liang H, Acharya G. Novel corona virus disease (COVID-19) in pregnancy: what clinical recommendations to follow? Acta Obstet Gynecol Scand 2020;99(04):439-442

5 Chen D, Yang H, Cao Y, et al. Expert consensus for managing pregnant women and neonates born to mothers with suspected or confirmed novel coronavirus (COVID-19) infection. Int J Gynaecol Obstet 2020;149(02):130-136

6 Chen H, Guo J, Wang C, et al. Clinical characteristics and intrauterine vertical transmission potential of COVID-19 infection in nine pregnant women: a retrospective review of medical records. Lancet 2020;395(10226):809-815

7 Chen S, Huang B, Luo DJ, et al. [Pregnant women with new coronavirus infection: a clinical characteristics and placental pathological analysis of three cases] (in Chinese). Zhonghua Bing Li Xue Za Zhi 2020;49(00):E005 (e-pub ahead of print)

8 Jiao J. Under the epidemic situation of COVID-19, should special attention to pregnant women be given? J Med Virol 2020 (e-pub ahead of print). Doi: $10.1002 /$ jmv. 25771

9 Poon LC, Yang H, Lee JCS, et al. ISUOG interim guidance on 2019 novel coronavirus infection during pregnancy and puerperium: information for healthcare professionals. Ultrasound Obstet Gynecol 2020. Doi: 10.1002/uog.22013

10 Schwartz DA. An Analysis of 38 pregnant women with COVID-19, their newborn infants, and maternal-fetal transmission of SARSCoV-2: maternal coronavirus infections and pregnancy outcomes. Arch Pathol Lab Med 2020 (e-pub ahead of print). Doi: 10.5858/ arpa.2020-0901-SA

11 Schwartz DA, Graham AL. Potential maternal and infant outcomes from (Wuhan) Coronavirus 2019-nCoV infecting pregnant women: lessons from SARS, MERS, and Other human coronavirus infections. Viruses 2020;12(02):194

12 Liu D, Li L, Wu X, et al. Pregnancy and perinatal outcomes of women with coronavirus disease (COVID-19) pneumonia: a preliminary analysis. AJR Am J Roentgenol 2020 (e-pub ahead of print). Doi: 10.2214/AJR.20.23072:1-6

13 Liu Y, Chen H, Tang K, Guo Y. Clinical manifestations and outcome of SARS-CoV-2 infection during pregnancy. J Infect 2020 (e-pub ahead of print). Doi: 10.1016/j.jinf.2020.02.028 
14 Liu H, Liu F, Li J, Zhang T, Wang D, Lan W. Clinical and CT imaging features of the COVID-19 pneumonia: Focus on pregnant women and children. J Infect 2020 (e-pub ahead of print). Doi: 10.1016/j. jinf.2020.03.007

15 Mullins E, Evans D, Viner RM, O'Brien P, Morris E. Coronavirus in pregnancy and delivery: rapid review. Ultrasound Obstet Gynecol 2020 (e-pub ahead of print). Doi: 10.1002/uog.22014

16 Panahi L, Amiri M, Pouy S. Risks of novel coronavirus disease (COVID-19) in pregnancy; a narrative review. Arch Acad Emerg Med 2020;8(01):e34

17 Zeng L, Xia S, Yuan W, et al. Neonatal early-onset infection with SARS-CoV-2 in 33 neonates born to mothers with COVID-19 in Wuhan, China. JAMA Pediatr 2020 (e-pub ahead of print). Doi: 10.1001/jamapediatrics.2020.0878

18 Zeng H, Xu C, Fan J, et al. Antibodies in infants born to mothers with COVID-19 pneumonia. JAMA 2020 (e-pub ahead of print). Doi: $10.1001 /$ jama.2020.4861

19 Fan C, Lei D, Fang C, et al. Perinatal transmission of COVID-19 associated SARS-CoV-2: should we worry? Clin Infect Dis 2020 (e-pub ahead of print). Doi: 10.1093/cid/ciaa226

20 Stower H. Lack of maternal-fetal SARS-CoV-2 transmission. Nat Med 2020;26(03):312

21 Qiao J. What are the risks of COVID-19 infection in pregnant women? Lancet 2020;395(10226):760-762

22 Centers for Disease Control. Interim infection prevention and control recommendations for patients with suspected or confirmed coronavirus disease 2019 (COVID-19) in healthcare settings. Available at: https://www.cdc.gov/coronavirus/2019-ncov/ hcp/infection-control-recommendations.html?CDC_AA_refVal= https\%3A\%2F\%2Fwww.cdc.gov\%2Fcoronavirus\%2F2019-ncov\%2 Finfection-control\%2Fcontrol-recommendations.html. Accessed April 14, 2020

23 Society for Maternal Fetal Medicine and Society for Obstetric and Anesthesia and Perinatology. Labor and Delivery COVID-19 Considerations. Available at: https://s3.amazonaws.com/cdn.smfm.org/media/2277/SMFM-SOAP_COVID_LD_Considerations_3-2720_(final)_PDF.pdf. Accessed April 1, 2020

24 Rosenbaum RA, Benyo JS, O'Connor RE, et al. Use of a portable forced air system to convert existing hospital space into a mass casualty isolation area. Ann Emerg Med 2004;44(06): 628-634

25 Bischoff W, Russell G, Willard E, Stehle J Jr. Impact of a novel mobile high-efficiency particulate air-ultraviolet air recirculation system on the bacterial air burden during routine care. Am J Infect Control 2019;47(08):1025-1027

26 Oren I, Haddad N, Finkelstein R, Rowe JM. Invasive pulmonary aspergillosis in neutropenic patients during hospital construction: before and after chemoprophylaxis and institution of HEPA filters. Am J Hematol 2001;66(04):257-262

27 Poston JT, Patel BK, Davis AM. Management of critically ill adults with COVID-19. JAMA 2020 (e-pub ahead of print). Doi: 10.1001/ jama.2020.4914

28 Grobman WA, Rice MM, Reddy UM, et al; Eunice Kennedy Shriver National Institute of Child Health and Human Development Maternal-Fetal Medicine Units Network. Labor induction versus expectant management in low-risk nulliparous women. $\mathrm{N}$ Engl J Med 2018;379(06):513-523

29 Barton JR, Sibai BM. Severe sepsis and septic shock in pregnancy. Obstet Gynecol 2012;120(03):689-706

30 Committee on Practice Bulletins-Obstetrics. ACOG practice bulletin no. 188: prelabor rupture of membranes. Obstet Gynecol 2018;131(01):e1-e14

31 Morris JM, Roberts CL, Bowen JR, et al; PPROMT Collaboration. Immediate delivery compared with expectant management after preterm pre-labour rupture of the membranes close to term (PPROMT trial): a randomised controlled trial. Lancet 2016;387 (10017):444-452
32 Mercer BM. Preterm premature rupture of the membranes. Obstet Gynecol 2003;101(01):178-193

33 Peaceman AM, Lai Y, Rouse DJ, et al; Eunice Kennedy Shriver National Institute of Child Health and Human Development Maternal-Fetal Medicine Units Network. Length of latency with preterm premature rupture of membranes before 32 weeks' gestation. Am J Perinatol 2015;32(01):57-62

34 Rouse DJ, Hirtz DG, Thom E, et al; Eunice Kennedy Shriver NICHD Maternal-Fetal Medicine Units Network. A randomized, controlled trial of magnesium sulfate for the prevention of cerebral palsy. N Engl J Med 2008;359(09):895-905

35 Pasquier JC, Claris O, Rabilloud M, et al. Intentional early delivery versus expectant management for preterm premature rupture of membranes at 28-32 weeks' gestation: a multicentre randomized controlled trial (MICADO STUDY). Eur J Obstet Gynecol Reprod Biol 2019;233:30-37

36 Cox SM, Leveno KJ. Intentional delivery versus expectant management with preterm ruptured membranes at 30-34 weeks' gestation. Obstet Gynecol 1995;86(06):875-879

37 Caughey AB, Cahill AG, Guise JM, Rouse DJ; American College of Obstetricians and Gynecologists (College); Society for MaternalFetal Medicine. Safe prevention of the primary cesarean delivery. Am J Obstet Gynecol 2014;210(03):179-193

38 Hui DS, Ip M, Tang JW, et al. Airflows around oxygen masks: A potential source of infection? Chest 2006;130(03):822-826

39 Hui DS, Hall S, Chan MT, Joynt GM, Chow B, Sung JJ. Risks posed by the use of oxygen therapy and non-invasive positive pressure ventilation: a pilot study. Hong Kong Med J 2009;15(Suppl 8):4-7

40 Somogyi R, Vesely AE, Azami T, et al. Dispersal of respiratory droplets with open vs closed oxygen delivery masks: implications for the transmission of severe acute respiratory syndrome. Chest 2004;125(03):1155-1157

41 Ankumah NE, Blackwell SC, Alrais MA, et al. Liberal versus indicated maternal oxygen supplementation in labor: a beforeand-after Trial. Am J Perinatol 2018;35(11):1057-1064

42 Jozwik M, Sledziewski A, Klubowicz Z, Zak J, Sajewska G, Pietrzycki $B$. [Use of oxygen therapy during labour and acid-base status in the newborn] (in Polish). Med Wieku Rozwoj 2000;4(04):403-411

43 Nesterenko TH, Acun C, Mohamed MA, et al. Is it a safe practice to administer oxygen during uncomplicated delivery: a randomized controlled trial? Early Hum Dev 2012;88(08):677-681

44 Qian G, Xu X, Chen L, et al. The effect of maternal low flow oxygen administration during the second stage of labour on umbilical cord artery pH: a randomised controlled trial. BJOG 2017;124(04):678-685

45 Thorp JA, Trobough T, Evans R, Hedrick J, Yeast JD. The effect of maternal oxygen administration during the second stage of labor on umbilical cord blood gas values: a randomized controlled prospective trial. Am J Obstet Gynecol 1995;172(2, Pt 1):465-474

46 American College of Obstetricians and Gynecologists; Committee on Practice Bulletins-Obstetrics. ACOG practice bulletin no. 127: management of preterm labor. Obstet Gynecol 2012;119(06):1308-1317

47 Dixon CL, Too G, Saade GR, Gyamfi-Bannerman C. Past and present: a review of antenatal corticosteroids and recommendations for late preterm birth steroids. Am J Perinatol 2018;35(13):1241-1250

48 Gyamfi-Bannerman C, Thom EA, Blackwell SC, et al; NICHD Maternal-Fetal Medicine Units Network. Antenatal betamethasone for women at risk for late preterm delivery. N Engl J Med 2016;374(14):1311-1320

49 Kamath-Rayne BD, Rozance PJ, Goldenberg RL, Jobe AH. Antenatal corticosteroids beyond 34 weeks gestation: what do we do now? Am J Obstet Gynecol 2016;215(04):423-430

50 Porto AM, Coutinho IC, Correia JB, Amorim MM. Effectiveness of antenatal corticosteroids in reducing respiratory disorders in late preterm infants: randomised clinical trial. BMJ 2011;342:d1696

51 Yinon Y, Haas J, Mazaki-Tovi S, et al. Should patients with documented fetal lung immaturity after 34 weeks of gestation be treated with steroids? Am J Obstet Gynecol 2012;207(03):222.e1-222.e4 
52 Crowther CA, Haslam RR, Hiller JE, Doyle LW, Robinson JS; Australasian Collaborative Trial of Repeat Doses of Steroids (ACTORDS) Study Group. Neonatal respiratory distress syndrome after repeat exposure to antenatal corticosteroids: a randomised controlled trial. Lancet 2006;367(9526):1913-1919

53 Garite TJ, Kurtzman J, Maurel K, Clark R; Obstetrix Collaborative Research Network. Impact of a 'rescue course' of antenatal corticosteroids: a multicenter randomized placebo-controlled trial. Am J Obstet Gynecol 2009;200(03):248.e1-248.e9

54 Lee MJ, Davies J, Guinn D, et al. Single versus weekly courses of antenatal corticosteroids in preterm premature rupture of membranes. Obstet Gynecol 2004;103(02):274-281

55 McEvoy C, Bowling S, Williamson K, et al. The effect of a single remote course versus weekly courses of antenatal corticosteroids on functional residual capacity in preterm infants: a randomized trial. Pediatrics 2002;110(2, Pt 1):280-284

56 Murphy KE, Hannah ME, Willan AR, et al; MACS Collaborative Group. Multiple courses of antenatal corticosteroids for preterm birth (MACS): a randomised controlled trial. Lancet 2008;372 (9656):2143-2151

57 Wapner RJ, Sorokin Y, Thom EA, et al; National Institute of Child Health and Human Development Maternal Fetal Medicine Units Network. Single versus weekly courses of antenatal corticosteroids: evaluation of safety and efficacy. Am J Obstet Gynecol 2006;195(03): 633-642

58 American College of Obstetricians and Gynecologists. ACOG Practice Bulletin no. 202: gestational hypertension and preeclampsia. Obstet Gynecol 2019;133(01):e1-e25
59 American College of Obstetricians and Gynecologists and Society of Maternal Fetal Medicine. Committee opinion no 652: magnesium sulfate use in obstetrics. Obstet Gynecol 2016;127(01): e52-e53

60 Altman D, Carroli G, Duley L, et al; Magpie Trial Collaboration Group. Do women with pre-eclampsia, and their babies, benefit from magnesium sulphate? The Magpie Trial: a randomised placebo-controlled trial. Lancet 2002;359(9321):1877-1890

61 Little P. Non-steroidal anti-inflammatory drugs and covid-19. BMJ 2020;368:m1185

62 Russell B, Moss C, George G, et al. Associations between immunesuppressive and stimulating drugs and novel COVID-19-a systematic review of current evidence. Ecancermedicalscience 2020; 14:1022

63 Voiriot G, Philippot Q, Elabbadi A, Elbim C, Chalumeau M, Fartoukh M. Risks related to the use of non-steroidal anti-inflammatory drugs in community-acquired pneumonia in adult and pediatric patients. J Clin Med 2019;8(06):E786

64 FitzGerald GA. Misguided drug advice for COVID-19. Science 2020;367(6485):1434

65 Varrassi G. Warning against the use of anti-inflammatory medicines to cure COVID-19: building castles in the air. Adv Ther 2020 (e-pub ahead of print). Doi: 10.1007/s12325-020-01321-1

66 Amici C, Di Caro A, Ciucci A, et al. Indomethacin has a potent antiviral activity against SARS coronavirus. Antivir Ther 2006;11 (08):1021-1030

67 Committee on Practice Bulletins-Obstetrics. Practice bulletin no. 183: postpartum hemorrhage. Obstet Gynecol 2017;130(04):e168-e186 